\title{
Estratégias organizacionais e inovações na gestão da Atenção Primária à Saúde no Brasil
}

A Atenção Primária à Saúde no Brasil vem passando por uma série de transformações desde seus primórdios com o então "Programa de Saúde da Família" em 1994 até a formulação em 2006 de uma política específica no seio do Ministério da Saúde e, mais recentemente, em 2019, com a institucionalização da Secretaria de Atenção Primária à Saúde (SAPS) no âmbito federal. Durante todo esse percurso, uma série de arranjos organizacionais foram desenvolvidos. Nesse caminhar, Tasca et al. ${ }^{1}$ ressaltam quatro conjuntos de inovações que mais recentemente vêm se destacando: (i) "teletrabalho", mediado por profissionais de saúde que vêm progressivamente se ampliando e se consolidando na rede de serviços com a nomenclatura "telemedicina/ telessaúde", (ii) fortalecimento das ações de formação profissional no âmbito do SUS, mediados por plataforma virtuais e novas modalidades em serviço multiprofissional como as Residências Multiprofissionais e os Mestrados Profissionais em Rede e convencionais, em Atenção Primária, (iii) resgate dos instrumentos já consagrados para avaliação em atenção primária e novos formatos/métodos de coleta de dados com o uso de tablets e coleta de dados remota, (iv) gestão, informação e novas formas de comunicação na APS com o uso de mídias sociais no apoio à gestão na atenção primária à saúde, tal como também relatado por Pinto e Rocha ${ }^{2}$. Essas informações não estão sistematizadas em muitos locais, porque frequentemente não podem ser padronizadas em dados quantitativos, formando uma base de dados no sentido mais narrativo e qualitativo.

Desses quatro tipos de inovações na APS, partindo do Centro-Oeste para as demais regiões brasileiras, este número temático traz um conjunto de contribuições para o debate no SUS, com novas perspectivas e abordagens, com destaque para Campo Grande. Essa cidade implementou uma série de medidas de apoio à gestão, formação profissional e desenvolvimento de pesquisas de avaliação, através do Laboratório de Inovação na Atenção Primária à Saúde (INOVAAPS) ${ }^{3}$, desenvolvidas com apoio da Fundação Oswaldo Cruz (https://labinovaapsfiocruz.com.br/portal/\#/).

A estratégia de um "território de ensino-aprendizagem" para a APS no SUS brasileiro pode ser adaptada e replicada em outras realidades locais no país, com apoio de instituições acadêmicas. Contudo, sabemos que dar continuidade à execução das ações propostas é uma tarefa árdua. Campo Grande e o Brasil necessitam manter um comprometimento estreito com a Estratégia Saúde da Família, de modo a dar-lhe continuidade e permanente aprimoramento, com ferramentas integradas de gestão, visando superar os desafios do cuidado qualificado em saúde com a centralidade nas demandas dos usuários e dos territórios. A pandemia por COVID-19 demonstrou ao mundo desde o início de 2020 e ao longo do período de imunização em 2021, que as ações e serviços de atenção primária e vigilância em saúde são estruturantes de qualquer sistema nacional de saúde que pretenda dar acesso e cuidado longitudinal de qualidade à sua população.

Daniel Ricardo Soranz (http://orcid.org/0000-0002-7224-5854) ${ }^{1}$

Roberto de Araújo Raposo (https://orcid.org/0000-0002-9652-9763) ${ }^{1}$

${ }^{1}$ Fundação Oswaldo Cruz. Rio de Janeiro RJ Brasil.

\section{Referências}

1. Tasca R, Massuda A, Carvalho WM, Buchweitz C, Harzheim E. Recomendações para o fortalecimento da atenção primária à saúde no Brasil. Rev Panam Salud Publica 2020; 44:e4.

2. Pinto LF, Rocha CMF. Inovações na Atenção Primária em Saúde: o uso de ferramentas de tecnologia de comunicação e informação para apoio à gestão local. Cien Saude Colet 2016; 21(5):1433-1448.

3. Centro de Estudos Estratégicos (CEE). Fundação Oswaldo Cruz (Fiocruz). Observatório de Tecnologias da Informação e Comunicação em Saúde de Campo Grande, Mato Grosso do Sul [Internet]. [acessado 2021 fev 11]. Disponível em: https:// labinovaapsfiocruz.com.br/portal/\#/. 